EPJ Web of Conferences 59, 15003 (2013)

DOI: $10.1051 /$ epjconf/20135915003

(C) Owned by the authors, published by EDP Sciences, 2013

\title{
Numerical simulations of energy transfer in two collisionless interpenetrating plasmas
}

\author{
S. Davis ${ }^{1}$, R. Capdessus ${ }^{1}$, E. d'Humières ${ }^{1}$, A.V. Brantov² ${ }^{2}$ S. Bochkarev² \\ and V. Tikhonchuk ${ }^{1}$ \\ ${ }^{1}$ Univ. Bordeaux - CNRS - CEA, CELIA, UMR 5107, 33405 Talence Cedex, France \\ 2 P.N. Lebedev Physics Institute, Russian Academy of Sciences, Moscow, Russia
}

\begin{abstract}
Ion stream instabilities are essential for collisionless shock formation as seen in astrophysics. Weakly relativistic shocks are considered as candidates for sources of high energy cosmic rays. Laboratory experiments may provide a better understanding of this phenomenon. High intensity short pulse laser systems are opening possibilities for efficient ion acceleration to high energies. Their collision with a secondary target could be used for collisionless shock formation. In this paper, using particle-in-cell simulations we are studying interaction of a sub-relativistic, laser created proton beam with a secondary gas target. We show that the ion bunch initiates strong electron heating accompanied by the Weibel-like filamentation and ion energy losses. The energy repartition between ions, electrons and magnetic fields are investigated. This yields insight on the processes occurring in the interstellar medium (ISM) and gamma-ray burst afterglows.
\end{abstract}

\section{INTRODUCTION}

Collisionless shocks are the ubiquitous phenomena in the Universe. Weakly relativistic shocks are created in the supernova explosions. It is believed that relativistic shocks are responsible for strong electron heating, magnetic field generation and subsequent emission of hard $\mathrm{X}$ and gamma rays in Gamma Ray Bursts (GRB). The collisionless shocks are considered to be the source of energy redistribution in Nature and high energy cosmic rays. However, a detailed mechanism of energy transformation of fast plasma flows into relativistic electrons and large amplitude magnetic fields is not known. The hypothesis of energy equipartition in a collisionless shock between the electrons, ions and magnetic fields has been shown in recent astrophysical models using large scale numerical simulations [1]. Laboratory experiments may provide further understanding of this obscure process. However, acceleration of sufficiently large volumes of matter to relativistic energies requires concentration of high densities of energy over a short time scale. Only high energy short pulse laser systems could be suitable for such experiments. The Target Normal Sheath Acceleration (TNSA) [2] can provide protons with energies of several tens of $\mathrm{MeV}$ corresponding to the streaming velocities of $20-30 \%$ of the velocity of light. The number of fast ions produced in one shot exceeds $10^{12}$, which should be sufficient for the collective processes to become significant. The range of such protons is of the order of $1 \mathrm{~g} / \mathrm{cm}^{2}$, so the collisional effects are not significant for distances of many ion inertia length, which define the characteristic thickness of the shock front.

It was theoretically predicted that collisionless shocks may occur at mildly relativistic streaming velocities with a fraction $\sim 10^{-4}$ of the total energy converted into magnetic field unless the electrons are heated significantly [3]. Experiments with non-relativistic laser pulses of sub-nanosecond duration and energy of a few hundred Joules demonstrate a possibility of plasma streams formation with high

This is an Open Access article distributed under the terms of the Creative Commons Attribution License 2.0, which permits unrestricted use, distribution, and reproduction in any medium, provided the original work is properly cited. 
Mach numbers, but the plasma temperature is relatively low and the ion mean free path remains shorter or comparable to the shock front thickness $[4,5]$. The magnetic field energy was not measured. Large scale numerical simulations $[6,7]$ demonstrate an important role that the Weibel instability plays in the energy transfer in streaming plasmas in astrophysical conditions.

In our previous paper [8] we proposed to collide the TNSA accelerated ion bunch with appropriately chosen low density plasma so that the collective effects will dominate the ion stopping. This paper is dedicated to developing a detailed model of electron heating and magnetic field generation in the overlapping region of the colliding plasmas: the first stage of formation of a collisionless shock.

The characteristics of the high energy proton bunch were optimized in large scale PIC simulations using the 2D Particle-in-Cell (PIC) code PICLS [9] and the 3D PIC code MANDOR [10]. The ions are accelerated from an over-dense target to sub-relativistic velocities by the TNSA mechanism. The simulations of the ion bunch collision with the secondary plasma were conducted with the code PICLS. The interaction takes place at the distance of $1 \mathrm{~mm}$ from the ion source and the time interval $t_{\max } \sim 4 \mathrm{ps}$ is defined by the length of the proton bunch. The choice of the distance is motivated by the necessity to protect the second plasma from undesired emission from the ion source and the divergence of the ion beam. We choose equal densities for both plasmas of $n_{0}=10^{18} \mathrm{~cm}^{-3}$ in order to optimise the interaction. This value is defined by the total number of accelerated ions. The plasma instabilities are excited by the streaming ions. The overall interaction time of about one ion plasma period, $\omega_{\mathrm{pi}} t_{\max } \sim 6$, is not sufficient for excitation of ion instabilities and full shock formation. Nevertheless, in this time interval one can see the development of electron instabilities, magnetic field generation and electron heating: the processes that are investigated in this paper.

\section{SIMULATION RESULTS}

\subsection{Summary of the simulation parameters}

For simulations of ion acceleration we chose a $1 \mu \mathrm{m}$ wavelength linearly polarized laser pulse with intensity of $10^{20} \mathrm{~W} / \mathrm{cm}^{2}$, pulse duration of $0.35 \mathrm{ps}$ and the energy of $3.5 \mathrm{~kJ}$. It interacts with a $5 \mu \mathrm{m}$ thick $\mathrm{CH}$ foil (density $4 \times 10^{22} \mathrm{~cm}^{-3}$ ) and accelerates electrons to the average energy of $16 \mathrm{MeV}$. The accelerated protons in the energy interval $10-30 \mathrm{MeV}$ have a divergence of a few degrees. Their density just after the acceleration is $\sim 10^{19} \mathrm{~cm}^{-3}$ and they are spread over the layer of a thickness of $\sim 10 \mu \mathrm{m}$. Assuming that they are accelerated from the spot of $110 \mu \mathrm{m}$ in diameter, we estimate the total number of protons to be $\sim 10^{13}$ in the source region. These protons spread ballistically along the propagation direction to the thickness of $\sim 100 \mu \mathrm{m}$ at a distance of $\sim 1 \mathrm{~mm}$ from the source.

These parameters are inputs for modeling of the proton bunch interaction with the secondary plasma. The simulations were conducted in the center of mass reference frame. The colliding plasmas have equal densities of $10^{18} \mathrm{~cm}^{-3}$ and equal bulk velocities $v_{i}=0.2 \mathrm{c}$. The target plasma moving to the left has initially the Maxwellian distributions of electrons and ions with the temperature of $100 \mathrm{eV}$. The plasma moving to the right plays the role of the external jet. The transverse ion temperature is $10 \mathrm{keV}$ and their parallel temperature is $10 \mathrm{MeV}$. The ion to electron mass ratio is 1836 . The main part of electrons has a Maxwellian distribution with the temperature of $10 \mathrm{keV}$, there is also a hot component with the parallel temperature of $2.5 \mathrm{MeV}$, transverse temperature $10 \mathrm{keV}$ and the relative density $n_{\mathrm{h}} / n_{0}=0.1$. This hot component represents the laser accelerated electrons that did not transfer their energy to ions. The simulation box length in the propagation direction is $1200 \mu \mathrm{m}$ and $300 \mu \mathrm{m}$ in the transverse direction. These lengths are comparable to the ion inertia length, $c / \omega_{\mathrm{pi}}=220 \mu \mathrm{m}$.

\subsection{Energy balance in the colliding plasmas}

At the initial moment in the center of mass reference frame, the energy is stored in the kinetic motion of the ions. The plasma moving to the right has twice the energy because of the parallel ion velocity 

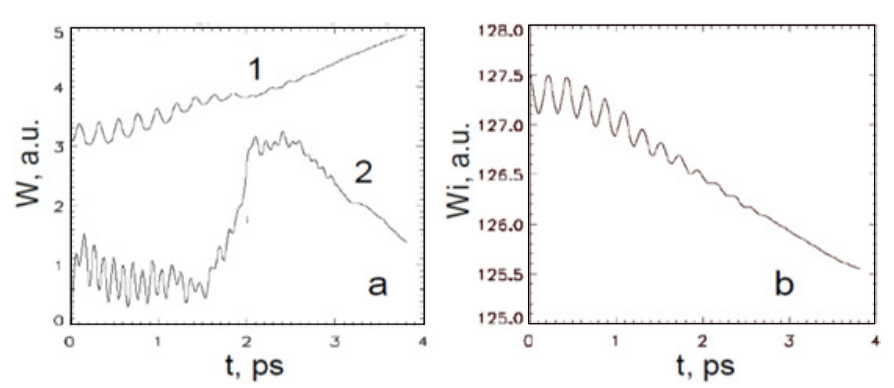

Figure 1. Time dependence of the electron ( $a, 1)$ and ion (b) energy in the simulation volume as well as the energy of the electromagnetic field $(a, 2)$. Energy units are the same for all curves.

spread. The electrons are carrying about 3\% of energy in the hot component. The electron contribution to the energy of the target plasma is negligible because of the large mass ratio. Figure 1 shows energy evolution in the computation volume with time. The electron, ion and magnetic field energies are shown separately. One can see two distinct phases of evolution. Up to $1.5 \mathrm{ps}$, the electron energy remains almost constant and the self-generated magnetic field remains at a relatively low level of about $1 \%$. The ion energy at this stage does not change. This stage corresponds to mixing of electron populations of two plasmas and their isotropization.

The second stage, $t>1.5 \mathrm{ps}$, is manifested by fast growth of the magnetic field energy accompanied by a progressive decrease of the ion energy. As our simulation is collisionless, this energy exchange indicates that ions are involved in the collective process. The characteristic time of the ion energy loss, $\mathrm{t}_{\text {ion }}=W_{\mathrm{i}}(0) /\left(d W_{\mathrm{i}} / d t\right) \sim 250 \omega_{\mathrm{pi}}^{-1}$, is of the same order as reported in [1]. Unfortunately, our simulation time was too short for more significant ion slowing down. After 3 ps the size of the overlap region begins to decrease and the magnetic field energy goes down. However, this demonstrates how energy exchange proceeds and the role of the magnetic field.

\subsection{Density profiles in the overlap region}

The density enhancement in the overlap region is the signature of ion slowing down and shock formation. Figure 2 shows the ion and electron density distributions in the simulation box. The overlap region at the time moment of $2.5 \mathrm{ps}$ is in the interval $x=500-700 \mu \mathrm{m}$. The cut in panel $\mathrm{c}$ shows that the amplitude of the filamentary structures is much larger than the sum of the densities of overlapping plasmas. A significant difference between the ion and electron density distributions indicates the charge separation in the filaments. The characteristic spacing between the filaments in the $y$-direction parallel to the shock front is of the order of 10-20 $\mu \mathrm{m}$, which is comparable to the electron inertia length, $c / \omega_{\mathrm{pe}}=5 \mu \mathrm{m}$. The length of filaments is of the order of $100 \mu \mathrm{m}$, much larger than their width. This fact, along with the magnetic field excitation shown in Fig. 3, indicates the electromagnetic nature of the instability. Most likely the electron Weibel instability is excited at the initial stage. Over time it evolves into the nonlinear stage: the electron density modulation creates the electrostatic field in the shock front plane, driving the transverse ion motion [11]. Thus, ions contribute to the nonlinear dynamics and energy transfer to electrons.

\subsection{Magnetic field generation}

The magnetic field is directed out of the simulation plane along the $z$ axis. This localization best identifies the instability zones and the dominant physical processes there. At the first stage of interaction, 


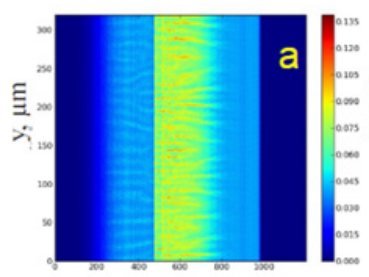

$\mathrm{x}, \mu \mathrm{m}$

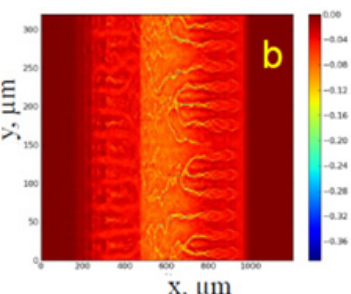

$\mathrm{x}, \mu \mathrm{m}$

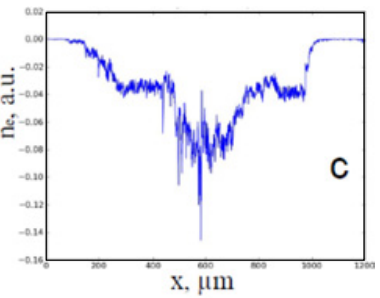

C

Figure 2. Ion (a) and electron (b) density distribution in the simulation box at $\mathrm{t}=2.5 \mathrm{ps}$. The overlap region extends from $x=500 \mu \mathrm{m}$ to $x=700 \mu \mathrm{m}$. The shock precursors are developing due to hot electrons ejected from the overlap region. Panel c presents the electron density cut at $\mathrm{y}=75 \mu \mathrm{m}$ showing a strong enhancement in the filament.
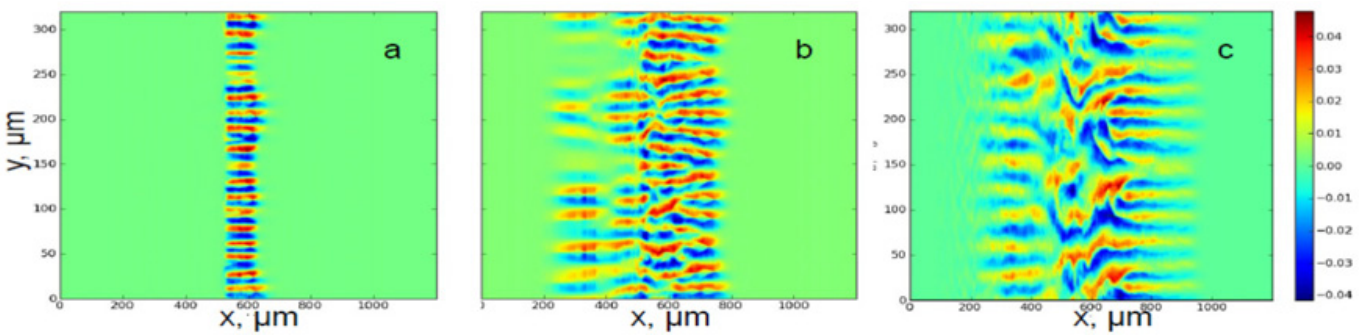

Figure 3. Magnetic field distribution in the simulation box at the time of 1.7 (a), 2.0 (b) and $2.5 \mathrm{ps}$ (c). The field amplitude 0.04 corresponds to the intensity of $4 \mathrm{MG}$.

$t<1.5 \mathrm{ps}$, the instability is localized in the overlap region and it is saturated at a high magnetic field level $\sim 3-4$ MG. The corresponding energy density is $\sim 10^{5} \mathrm{~J} / \mathrm{cm}^{3}$. This is comparable to the electron energy density: $\sim$ few per cent of the ion kinetic energy density $n_{i} m_{i} v_{i}^{2}$.

The intensity of magnetic field does not increase at the second stage of the interaction. Here, it spreads very quickly with energetic electrons in the upstream regions creating large amplitude precursors. Concomitantly, nonlinear evolution in the downstream region leads to merging of filaments and formation of large scale vortices. The mechanism of the precursor formation needs to be further elucidated. It could slow down the ions even before entering in the shock. The high magnetic pressure in filaments leads to the strong charge separation fields of the order of $10^{11} \mathrm{~V} / \mathrm{m}$ that could be responsible for the fast ion to electron energy exchange.

\section{CONCLUSIONS}

Laser accelerated bunches of $10-30 \mathrm{MeV}$ protons carrying energy of a few tens of Joules can be used for studies of the collisionless energy transfer between the ions and electrons. Although the bunch energy is not sufficient for full shock formation, it is possible to create the conditions for excitation of electron Weibel instability in a few ps time scale with subsequent magnetic field generation in the filaments, electron heating and acceleration. This corresponds to significant ion energy losses. The estimated ion energy loss length $v_{\mathrm{i}} t_{\mathrm{ion}} \approx 1.5 \mathrm{~cm}$ is four orders of magnitude smaller than the collisional stopping length. If confirmed in larger scale numerical simulations, this process could be an obstacle for the ion fast ignition schemes. These in situ laboratory collisionless shocks will yield insights into astrophysical processes: e.g, GRB external jet interaction with the ISM. 


\section{IFSA 2011}

\section{References}

[1] A. Spitkovsky, Astrophys. J. 673, L39 (2008)

[2] R. A. Snavely et al., Phys. Rev. Lett. 85, 2945 (2000)

[3] T. Kato \& H. Takabe, Astrophys. J. 681, L93 (2008)

[4] Y. Kuramitsu et al., Phys. Rev. Lett. 106, 175002 (2011)

[5] V.T. Tikhonchuk et al., Plasma Phys. Control. Fusion 50, 124056 (2008)

[6] R. Fonseca et al., Phys. Plasmas 10, 1979 (2003)

[7] R.A. Fonseca et al., Plasma Phys. Control. Fusion 50, 124034 (2008)

[8] S. P. Davis et al. J. Phys.: Conf. Ser. 244, 04206 (2010)

[9] Y. Sentoku \& A. J. Kemp, J. Comp. Phys. 227, 6846 (2008)

[10] D. V. Romanov et al., Phys. Rev. Lett. 93, 215004 (2004)

[11] M. Tzoufras et al., Phys. Rev. Lett. 96, 105002 (2006) 